\title{
Arduino: uma proposta para o ensino Introdutório de programação $\mathbf{C} / \mathbf{C}++$
}

\section{Arduino: a proposal for teaching Introductory teaching of $\mathrm{C} / \mathrm{C}++$ programming}

DOI: $10.46814 /$ lajdv3n4-038

Recebimento dos originais: 01/05/2021

Aceitação para publicação: 31/06/2021

\author{
Allison Lopes de Oliveira \\ Mestre em Engenharia Elétrica \\ Instituto Federal do Triangulo Mineiro - Campus Paracatu \\ Rodovia Alírio Herval (MG 188) - KM 167 \\ Bairro: Paracatuzinho \\ E-mail: allissonoliveira@iftm.edu.br \\ Willian Antonio Gonçalves \\ Mestre em Tecnologias da Informação Aplicadas à Biologia Computacional \\ Instituto Federal do Norte de Minas Gerais - Campus Arinos \\ Rodovia MG 202, Km 407 \\ CEP: $38.680-000$ - Arinos/MG \\ E-mail: willian.goncalves@ifnmg.edu.br \\ Raphael Magalhães Hoed \\ Mestre em Computação Aplicada \\ Instituto Federal do Norte de Minas Gerais - Campus Januária \\ Fazenda São Geraldo, S/N Km 06 \\ CEP: 39480-000 - Januária/MG \\ E-mail: raphael.hoed@ifnmg.edu.br
}

\section{RESUMO}

Este artigo apresenta uma proposta diferenciada de uma nova metodologia de ensino, apoiada por ferramentas computacionais que podem contribuir para a melhoria do aprendizado dos alunos do Curso Técnico em Informática do Instituto Federal do Norte de Minas Gerais (IFNMG), Campus Arinos. As dificuldades encontradas pelos alunos na elaboração de um raciocínio estruturado para a solução de um problema computacional, de compreensão de conceitos, e a complexidade dos ambientes de desenvolvimento são, frequentemente, obstáculos ao aprendizado. Tais problemas podem ser amenizados pelo uso didático de novas plataformas, construídas para facilitar à aprendizagem pela experimentação. Com o intuito de tornar as aulas atrativas, objetivas e com o foco no aluno, pretendese aplicar a Plataforma Arduino que é uma excelente ferramenta para o ensino de conceitos de Lógica de Programação, além de ser um vasto repositório de recursos para experimentação prática, e um laboratório de baixo custo para testes de programas em $\mathrm{C} / \mathrm{C}++$. O Arduino une essas ferramentas, resultando num ambiente de aprendizado fácil, com recursos de interfaceamento para sensores e atuadores. A aplicação coordenada do Arduino, no contexto da disciplina Algoritmos, pode conduzir o aluno a um aprendizado facilitado, consistente e voltado a aplicações práticas.

Palavras-chave: Metodologia de Ensino, Programação, Algoritmos, Arduino. 


\section{ABSTRACT}

This article presents a differentiated proposal of a new teaching methodology, supported by computational tools that can contribute to the improvement of the learning process of students of the Technical Course in Computer Science of the Federal Institute of Northern Minas Gerais (IFNMG), Arinos Campus. The difficulties faced by students in developing a structured reasoning to solve a computational problem, understanding concepts, and the complexity of development environments are often obstacles to learning. These problems can be alleviated by the didactic use of new platforms, built to facilitate learning through experimentation. In order to make the classes attractive, objective and student focused, we intend to apply the Arduino platform, which is an excellent tool for teaching Logic Programming concepts, besides being a vast repository of resources for practical experimentation, and a low cost laboratory for testing $\mathrm{C} / \mathrm{C}++$ programs. Arduino brings these tools together, resulting in an easy learning environment with interfacing capabilities for sensors and actuators. The coordinated application of Arduino, in the context of the Algorithms course, can lead the student to a facilitated, consistent, and practical learning experience.

Keywords: Teaching Methodology, Programming, Algorithms, Arduino.

\section{INTRODUÇÃO}

O curso Técnico em Informática é dividido em três módulos (anuais) e visa a formação técnicaprofissional do aluno. O egresso será capaz de compreender o funcionamento do computador, as suas possibilidades de configuração, o desenvolvimento de programas (software), implementar e manter uma rede de computadores e sua integração com outras áreas.

As atenções desta pesquisa voltam-se, especificamente, para a disciplina algoritmos, ofertada no primeiro módulo do curso, no turno matutino e vespertino para os alunos matriculados no $1^{\circ}$ do Ensino Médio Integrado. A referida disciplina tem como propósito capacitar o aluno para os conceitos básicos de programação. De acordo com Cechinel (2008), as dificuldades inerentes ao processo de ensino-aprendizagem de algoritmos e programação estão relacionadas a diversos fatores e são objeto de estudo em inúmeros trabalhos. Como exemplo, o autor destaca que:

\footnotetext{
"Smith (1981) aponta as dificuldades que os acadêmicos encontram em relacionar os significados das informações recebidas e as suas dependências com outros conteúdos; Falkembach et al.(2003) relatam a existência de dificuldades no entendimento dos enunciados dos problemas, e Olsen (2005) fala sobre a falta de habilidade na construção de solução para os problemas apresentados."
}

\section{REFERENCIAL TEÓRICO}

O processo educacional, segundo Kenski (1999 apud Trevelin, 2013), é predominantemente semipresencial uma vez que é impossível se pensar que todas as atividades educativas conducentes ao conhecimento, ainda que previstas venham a ocorrer exclusivamente no espaço da escola, em sala de aula e diante da figura do professor. 
Na visão de Trevelin (2013), as tecnologias ampliam possibilidades de ensino para além do curto e delimitado espaço de presença física de professores e alunos em uma sala de aula e, ainda segundo Kenski (1999 apud Trevelin, 2013) a possibilidade de interação entre professores, alunos, pessoas, objetos e informações que estejam envolvidos no processo redefine toda a dinâmica de aula e cria diferentes vínculos entre seus participantes, fato este que merece atenção do professor.

De acordo com Trevelin (2013), ocorre que, conforme aponta Martins (1991 apud Trevelin, 2013), as novas tecnologias da informação interferem diretamente tanto para a prática docente como para o processo de aprendizagem o que gera a necessidade de adaptação contínua por parte dos aprendizes e dos próprios professores para acompanhar as inúmeras mudanças.

\subsection{A PLATAFORMA ARDUINO}

O Arduino é descrito pelos seus construtores como "uma plataforma de prototipação de fonte aberta, baseada em hardware e software fácil de utilizar. É planejada para artistas, designers, hobbystas e qualquer um interessado em criar ambientes ou objetos iterativos" (ARDUINO, 2011). As suas características, e uma ativa comunidade na internet, lhe têm garantido um forte crescimento na sua base de usuários.

O site do Arduino, em http://arduino.cc oferece um excelente suporte ao sistema, com seções de referência, e inclui uma seção denominada "Playground", em que conceitos de programação e de circuitos são explicados de forma simplificada.

\subsubsection{Hardware e Ambiente de Desenvolvimento do Arduino}

O projeto da placa é bastante intuitivo e a conexão dos pinos do microcontrolador vem disposta de uma forma bem simples. As placas Arduino permitem a utilização de módulos periféricos, conhecidos como "shields", que de acordo com Camargo et al. (2012) vem do termo inglês, "escudo", o que permite que estas placas tenham uma variedade de aplicações.

A Figura 1 mostra uma placa de Arduino Uno, exibindo suas diversas conexões para módulos periféricos e/ou para os mais diversos sensores. 
Figura 1 - Arduino Uno. Disponível em : <https://www.techtudo.com.br/noticias/noticia/20 13/10/o-que-e-um-arduino-e-o-que-pode-ser-feito-com-ele.html $>$

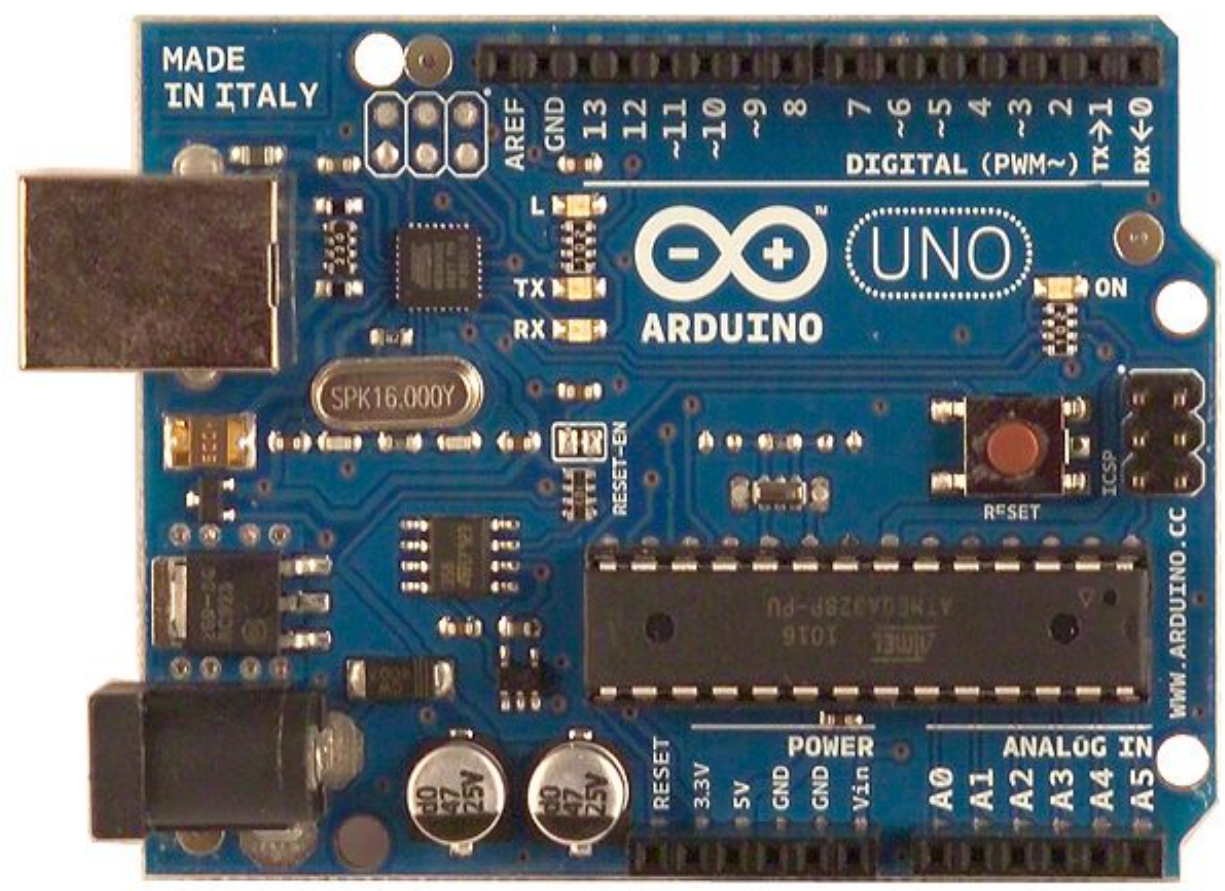

O ambiente de desenvolvimento do Arduino oferece uma interface de usuário simplificada não exigindo muitos conhecimentos de programação ou eletrônica. Sobre o arduino uno Barbosa (2013) informa que:

"É uma placa de microcontrolador baseado no ATmega328 (datasheet). Possui 14 entradas / saídas digitais (dos quais 6 podem ser usados como saídas PWM), 6 entradas analógicas, um cristal oscilador de $16 \mathrm{MHz}$, uma conexão USB, um conector de alimentação, um cabeçalho ICSP, e um botão de reset."

A Figura 2 mostra um algoritmo para fazer piscar um led ligado no pino digital 13 e, ainda com vários comentários sobre as funções de cada parte do código, prática de muitos tutoriais na internet para ajudar iniciantes. 
Figura 2 - Implementação de um exemplo simples no Arduino. Disponível em: http://www.abenge.org.br/cobenge/legado/arquivos/8/sessoestec/art1886.pdf

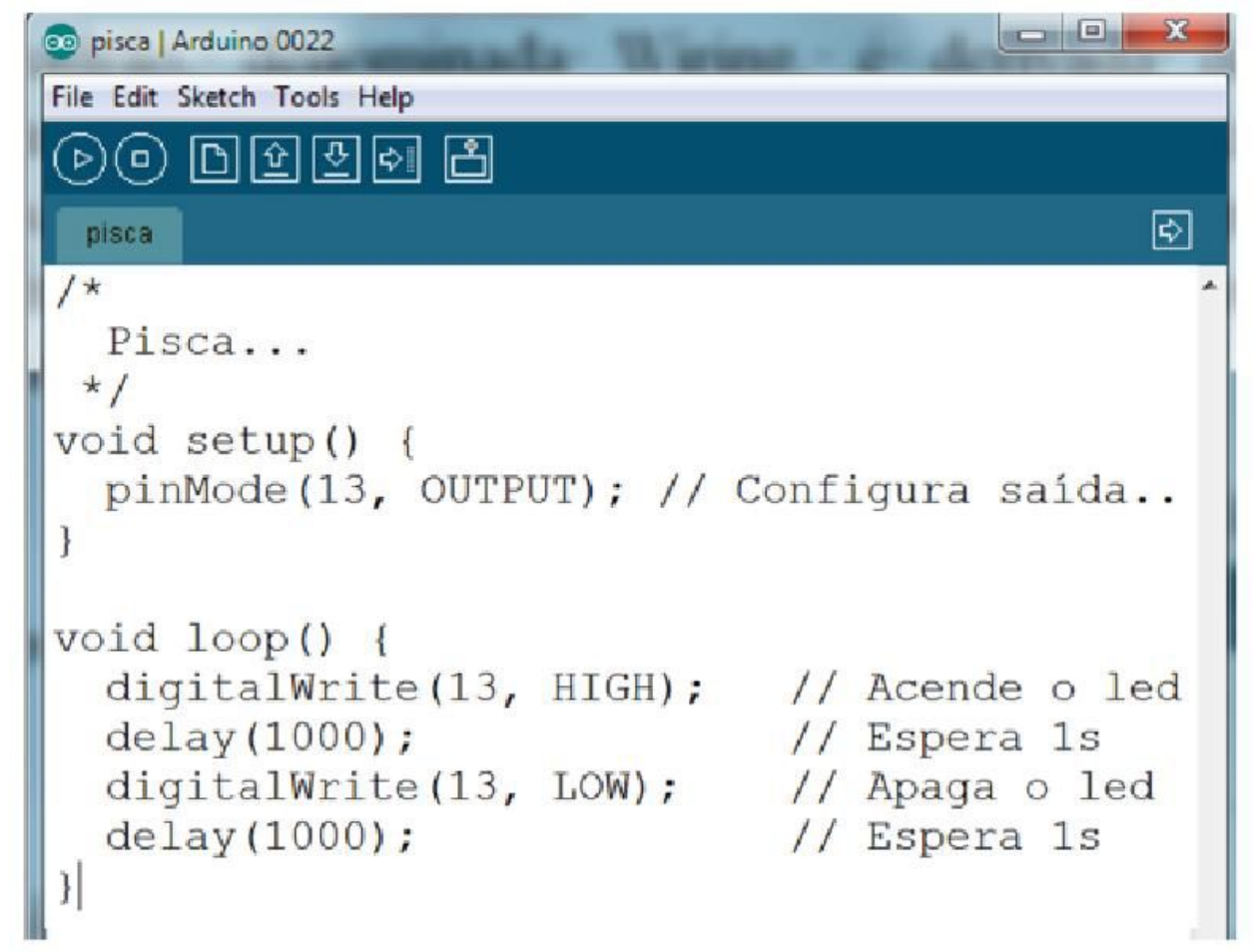

\section{METODOLOGIA}

Na primeira etapa deste trabalho, buscou-se conhecer os recursos da Plataforma Arduino. Para isso, um dos autores participou do curso de "Arduino", ofertado pelo UFG(Universidade Federal de Goiás), com carga horária de 8 horas. Neste curso foi possível familiarizar-se com a Plataforma Arduino.

$\mathrm{Na}$ segunda etapa deste trabalho o professor da disciplina e os autores deste projeto realizaram a divisão da turma em 5 grupos de 6 alunos, cada grupo tem um líder eleito por votação do grupo. Todos os grupos tem um acompanhamento dos autores do projeto.

A terceira etapa deste trabalho encontra-se em desenvolvimento. O seu propósito é planejar e elaborar projetos que abordem a utilização de uma grande variedade de recursos do Arduino de forma que o aluno possa ir além do currículo básico da disciplina de Algoritmos. De acordo com Bennet et al (2012), o processo de implantação e uso deste modelo pode ser algo não tão fácil de realizar, uma vez que não existem modelos definidos para tal. Porém, em sua experiência, a efetiva utilização do modelo deve possuir várias das seguintes características: as discussões são levadas pelos alunos para a sala de aula; essas discussões geralmente atingem ordens superiores de pensamento crítico; o trabalho colaborativo ocorre entre os alunos em função de várias discussões simultâneas; estudantes desafiam uns aos outros durante a aula, em função do conhecimento adquirido; os estudantes têm a posse do 
material; os estudantes transformam-se de ouvintes passivos para os alunos ativos no processo de ensino-aprendizagem. Este projeto teve início no $1^{\circ}$ trimestre de 2014. Naturalmente, em sala de aula, o professor terá um tempo maior para desenvolver atividades práticas com os alunos, em forma de projetos ou listas de exercícios direcionadas.

Com essa abordagem, pretende-se motivar o aluno em atividades de auto estudo com base em livros, materiais em hipertextos e hiperlinks, vídeos e outros recursos midiáticos. Consequentemente, como afirma Schneider (2013), espera-se que o aluno, dedique-se, a aquisição de seu próprio conhecimento e ao tempo necessário a atividade de acordo com suas características de aprendizagem. Em etapa subsequente, o professor da disciplina estimula o aluno a pesquisa e a interação com os colegas com o uso de ferramentas de tecnologia da informação.

\subsection{AVALIAÇÃO}

A avaliação será formada pelos seguintes itens:

Auto avaliação do aluno: Serão constituídas do conhecimento adquirido, habilidades, reconhecimento de responsabilidades. Avaliação por pares: Os membros dos grupos avaliarão seus colegas para verificar o desempenho e compromisso ao final de cada projeto. Avaliação do professor: Identificar participação, atitudes, habilidades, conhecimento e progressos de cada aluno e grupo. A nota será formada pelos resultados da avaliação e da Prova Teórica da disciplina. A prova prática será o resultado dos problemas devidamente registrados e apresentados.

\section{RESULTADOS E DISCUSSÕES}

Este trabalho se encontra em andamento e por isso não foi possível tabular dados parciais ou finais. Como experimento, durante dez aulas foi apresentada a Plataforma Arduino e exemplos de projetos nesta plataforma. Esta ação teve o intuito de perceber a reação dos alunos diante do primeiro contato com a nova ferramenta. E, apesar de informal, o retorno foi satisfatório! Os alunos ficaram motivados e determinados a criarem novos projetos.

\section{CONSIDERAÇÕES FINAIS}

De acordo com TechSmith (2013) as atividades complementares propostas pelo professor, ou seja, as "tarefas", no modelo Flipped Classroom são realizadas em sala de aula, em equipes, com o suporte do professor. Assim, os estudantes têm a oportunidade de solucionar suas dúvidas no momento em que elas ocorrem, com a ajuda de seus pares e do professor, o que promove um ambiente colaborativo de aprendizagem. Para Schneider (2013), na organização do Flipped Classroom, os níveis mais básicos da aprendizagem ocorrem por meio do estudo individualizado, permitindo que se 
organizem as atividades colaborativas (presenciais ou à distância) com o objetivo de criar conhecimento novo, por meio da discussão, da aplicação do conhecimento em situações-problema novas, que exijam o uso crítico e criativo dos conteúdos estudados. Ou seja, espera-se no futuro deste trabalho comprovar que a implementação do modelo do Flipped Classroom com base na utilização de OAs, criados para a disciplina Técnicas de Programação, demonstre que o processo de ensinoaprendizado dos alunos foi melhor que o processo tradicional, centralizado no professor. 


\section{REFERÊNCIAS}

ARDUINO. ARDUINO. Disponível em <http://arduino.cc>. Acesso em 18 abril. 2014.

BARBOSA, J. W. Sistema de Irrigação Automatizado utilizando a plataforma Arduino. Fundação Educacional do Município de Assis - FEMA - Assis, 2013. Disponível em: <https:/

/cepein.femanet.com.br/BDigital/arqTccs/1011330043.pdf>. Acesso em: abr. 2014.

BARSEGHIAN, T. Three Trends That Define the Future of Teaching and Learning. Disponível em $<$ http://blogs.kqed.org/mindshift/2011/02/three-trends-that-definethe-future-of-teaching-andlearning/>. Acesso em: abr. 2014.

BENNET, B. et al. The Flipped Class: What Does a Good One Look Like?. Disponível em: $<$ http://www.thedailyriff.com/articles/the-flipped-class-what-does-agood-one-look-like-692. php>. Acesso em: abr. 2014.

BERGMANN, J.; OVERMYER, J.; WILIE, B. The Flipped Class: What It Is and What It Is Not. Disponível em: <http://www.thedailyriff.com/articles/the-flipped-classconversation-689.php $>$. Acesso em: abr. 2014.

CAMARGO, C. B., COSTA, L. F., PARADISO,S. R. Sistema de Controle para Aquariolia. Marigá, 2012. Anais eletrônico - VI Mostra Interna de Trabalho de Iniciação Científica, 2012.

CECHINEL, C. et al. Desenvolvimento de Objetos de Aprendizagem para o Apoio à Disciplina de Algoritmos e Programação. In: XIX Simpósio Brasileiro de Informática na Educação - Workshop Ambientes de Apoio à Aprendizagem de Algoritmos e Programação, 2008, Fortaleza. Anais do Workshop Ambientes de Apoio à Aprendizagem de Algoritmos e Programação - XIX Simpósio Brasileiro de Informática na Educação, 2008. Disponível em: $<$ http://sbie2008.virtual.ufc.br/CD_ROM_COMPLETO/workshops/workshop\%202/Desenvol vimento $\% 20 \mathrm{de} \% 20$ Objetos $\% 20 \mathrm{de} \% 20$ Aprendizagem $\% 20$ para $\% 20 \mathrm{o} \% 20$ Apoio.pdf $>$. Acesso em: dez. 2013.

COLENCI-TREVELIN, A; PEREIRA, M. A. A.; OlIVEIRA NETO, J. D. A Utilização da Sala de Aula Invertida em Cursos Superiores de Tecnologia: Comparação entre o Modelo Tradicional e o Modelo Invertido "Flipped Classroom" adaptado aos Estilos de Aprendizagem. Revista de Estilos de Aprendizaje, $\quad$ v. 11, p. 137-150, 2013. Disponível em: $<$ http://learningstyles.uvu.edu/index.php/jls/article/view/12>. Acesso em: mar. 2014.

SCHNEIDER, E. I. et al. Sala de Aula Invertida em EAD: uma proposta de Blended Learning. Intersaberes (Facinter), v. 08, p. 68-81, 2013. Disponível em: $<$ http://www.grupouninter.com.br/intersaberes/index.php/revista/article/view/499>. Acesso em: mar. 2014.

TAROUCO, L. M. R.; FABRE, M. J. M.; TAMUSIUNAS, F. R. Reusabilidade de objetos educacionais. In: RENOTE (Revista Eletrônica de Novas Tecnologias na Educação). Porto Alegre: s.ed., v.1, n.1, fevereiro de 2003. Disponível em <http://www.nuted.ufrgs.br/oficinas /criacao/marie_reusabilidade.pdf $>$. Acesso em: abr. 2014.

TAROUCO, L. M. R. et al. Objetos de Aprendizagem para M-Learning. In: Congresso Nacional de Tecnologia da Informação e Comunicação, 2004, Florianópolis. Congresso Nacional de Tecnologia da 
Informação e Comunicação, 2004. v. $1 . \quad$ Disponível em: $<$ http://www.cinted.ufrgs.br/CESTA/objetosdeaprendizagem_sucesu.pdf $>$. Acesso em: mar. 2013.

TECHSMITH. Teachers Use Technology to Flip Their Classrooms. Disponível em: $<$ http://www.techsmith.com/flipped-classroom.html>. Acesso em: abr. 2014.

TUCKER, B. The Flipped Classroom. Disponível em: <http://educationnext.org/theflippedclassroom/>. Acesso em: abr. 2014.

VICARI, R. M. Linux Educacional - Módulo 5. Porto Alegre, 2010, 44 p. Apostila do Curso Linux Educacional - Universidade Federal do Rio Grande do Sul. Disponível em: $<$ http://webeduc.mec.gov.br/linuxeducacional/curso_le/pdf/baixar_para_impressao_todos_modul os.pdf $>$. Acesso em: mar. 2014. 